\title{
Samarkand in der Bundeshauptstadt - ein Ausstellungssaal im neo-timuridischen Stil für die Sammlung Henri Mosers im Bernischen Historischen Museum
}

\author{
Kaufmann, Katrin
}

DOI: https://doi.org/10.1515/9783110588330-008

Posted at the Zurich Open Repository and Archive, University of Zurich ZORA URL: https://doi.org/10.5167/uzh-181284

Book Section

Published Version

Originally published at:

Kaufmann, Katrin (2019). Samarkand in der Bundeshauptstadt - ein Ausstellungssaal im neo-timuridischen Stil für die Sammlung Henri Mosers im Bernischen Historischen Museum. In: Giese, Francine; el-Wakil, Leïla; Varela Braga, Ariane. Der Orient in der Schweiz: neo-islamische Architektur und Interieurs des 19. und 20. Jahrhunderts = L'orient en Suisse: architecture et intérieurs néo-islamiques des $19 \mathrm{e}$ et $20 \mathrm{e}$ siècles. Berlin: De Gruyter, 142-162.

DOI: https://doi.org/10.1515/9783110588330-008 


\title{
Samarkand in der Bundeshauptstadt - ein Ausstellungssaal im neo-timuridischen Stil für die Sammlung Henri Mosers im Bernischen Historischen Museum
}

\begin{abstract}
À force d'admirer nous tombons de fatigue. Un dernier effort nous conduit sur le minaret de Chir-Dar; c'est le ,bouquet', le dernier souvenir à emporter de Samarcande l'incomparable. Le panorama dont on jouit de là défie toute description [...]. Sauf l'aspect de Naples, des Camaldoli, le panorama de la Scheideck et la vue du Bosphore, je ne trouve, dans les souvenirs de mes nombreux voyages, rien qui m'ait laissé une pareille impression. Tous ces édifices de Samarcande, construits par des artistes persans, impressionnent vivement le visiteur par leurs dimensions grandioses et leur couleur merveilleuse. ${ }^{1}$
\end{abstract}

Im Mai 1922 wurde das Bernische Historische Museum (BHM) nach einer längeren Schliessung wiedereröffnet. Im obersten Stockwerk eines Anbaus war nun die Orientalische Sammlung Henri Moser Charlottenfels zu sehen, eine der weltweit grössten Sammlungen islamischen Kunstgewerbes, die Henri Moser (18441923) über mehrere Jahrzehnte hinweg zusammengetragen hatte. ${ }^{2}$

Als einziger Sohn des Schweizer Uhrenunternehmers Heinrich Moser in Sankt Petersburg geboren, wuchs Henri Moser zunächst in der Schweiz auf. ${ }^{3}$ Er ist vierundzwanzig Jahre alt, als er 1868 erstmals nach Zentralasien reist. Als Forschungsreisender, Jäger und Diplomat führt er ein oft abenteuerliches Leben, bevor er sich als Sammler und Ausstellungsmacher einen Namen macht. ${ }^{4}$ 1907 lässt er sich auf Schloss Charlottenfels in Neuhausen am Rheinfall nieder, der villenartigen Familienresidenz, um sich gänzlich seiner Orientalischen Sammlung widmen zu können. Diese vermacht Moser 1914 dem BHM als Stiftung, allerdings mit der Auflage, sie in „eigens dafür zu bestimmenden Räumen zweckmässig und würdig auszustellen". ${ }^{5}$

1 Moser 1886 [1885], 119.

2 In der Folge wird hierfür auch die kürzere Bezeichnung „Orientalische Sammlung“ verwendet.

3 Zu Mosers Biographie siehe Balsiger/Kläy 1992, 11-63, 203-220.

$4 \mathrm{Zu}$ Mosers Sammlung und seiner Tätigkeit als Ausstellungsmacher siehe Zeller 1915; 1922; 1923; Pfaff 1985; Kläy 1991; Balsiger/Kläy 1992; Kläy 1994; Hitzel 2016.

5 Stiftungsurkunde Orientalische Sammlung Henri Moser Charlottenfels im bernischen historischen Museum vom 26.1.1914 (BHM, Inv. 1915.670.0159). Laut der Stiftungsurkunde hatte die Unterbringung der Sammlung auf Kosten des Museums zu geschehen. Moser war für die Aufstellung der Sammlung einzubeziehen und verpflichtete sich, „allfällige von ihm gehegte besondere Wünsche für die Installation, die das Budget des bernischen historischen Museums 


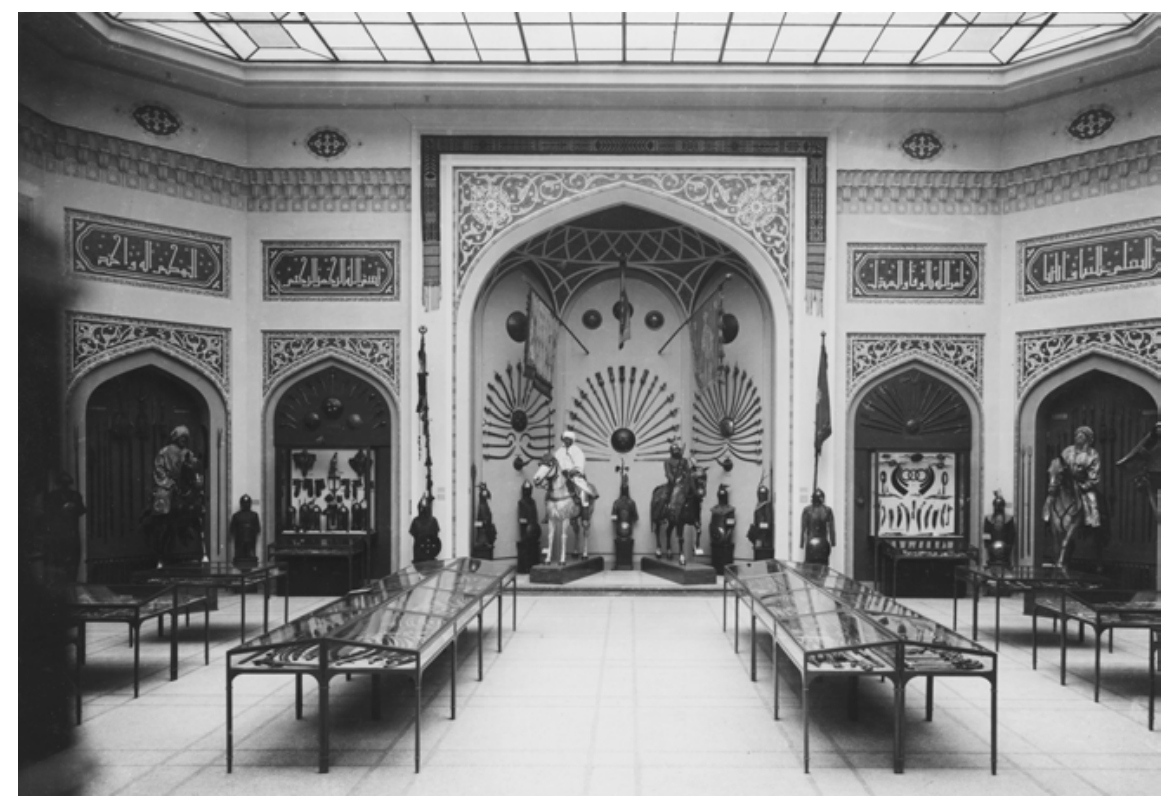

Abb. 1: Bern, Blick in den Waffensaal der Moser-Sammlung am BHM, um 1925. Bernisches Historisches Museum, Bern.

Die Ausstellung umfasste fünf Räume, wobei ein grosser Waffensaal das Prunkstück ausmachte (Abb. 1). Die Eingangssituation beschrieb die Gazette de Lausanne anlässlich der Eröffnung wie folgt:

Dès qu'on a franchi le seuil du Musée historique, on aperçoit en face de soi une grande et haute porte à deux vantaux dont l'encadrement rouge, bleu, jaune, rappelle le style oriental et nous prépare d'avance à ce que nous allons voir d'antique et d'exotique à la fois. ${ }^{6}$

Dieser Dekor im „style oriental“ war auch für die Ausstellungsräume verbindlich. Als Vorbild sollte auf Mosers ausdrücklichen Wunsch hin ein timuridisches Bauwerk dienen, das Gur-i Amir in Samarkand (Abb. 2). ${ }^{7}$ Neben der aussergewöhnlichen Sammlung islamischer Kunstgegenstände hatte das BHM nun auch die schweizweit einzigen Interieurs im neo-timuridischen Stil vorzuweisen.

übersteigen, auf eigene Kosten zu erfüllen“. Moser hatte lange nach der geeigneten Institution gesucht: Das Landesmuseum in Zürich sowie das Musée d'ethnographie in Genf hatten kein Interesse an der Sammlung. Auch die Verhandlungen mit Kanton und Stadt Schaffhausen führten zu keinem Resultat, siehe Kläy 1994, 343-344.

6 Gazette de Lausanne, 6.5.1922.

7 Siehe Bernisches Historisches Museum 1918, 6. Zudem: Briefe Moser an Saladin, 15.2.1918; 20.3.1918 (BHM). Zur Quellenlage siehe Anm. 8. 


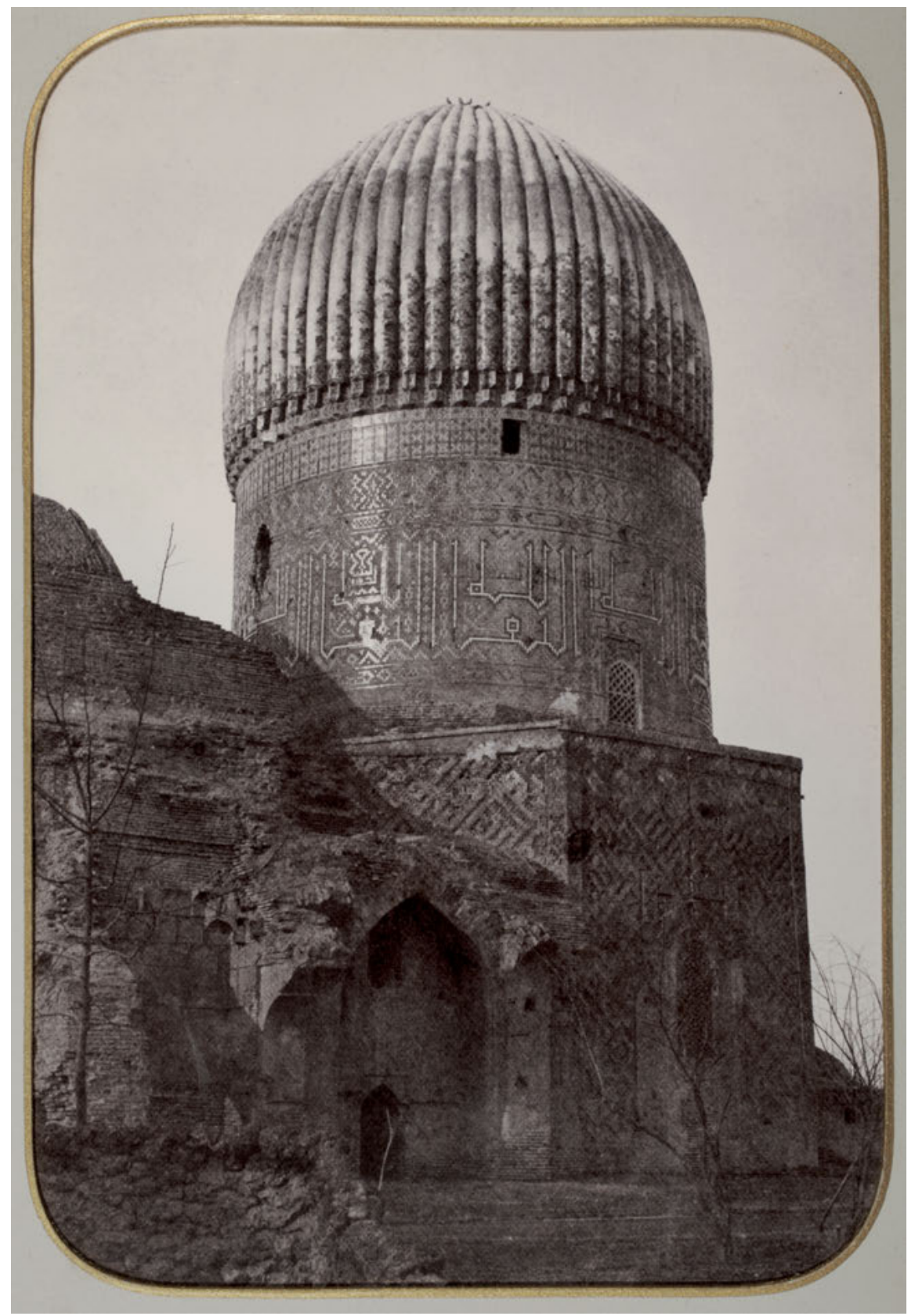

Abb. 2: Henri Moser, Turm und Kuppel des Gour Emir, 1889/90. Bernisches Historisches Museum, Bern, Inv. PH1.240.06584.01. 
Der vorliegende Aufsatz zeichnet anhand verschiedener Archivmaterialien aus der Zeit von 1914-1922 zunächst chronologisch auf, wie die Planungen zur Unterbringung der Moser-Sammlung verliefen. ${ }^{8}$ Im zweiten Teil wird der Fokus auf die Konzeption und Ausgestaltung des noch heute erhaltenen Waffensaals gelegt.

\section{Ein Erweiterungsbau für die Orientalische Sammlung und die Gestaltung der Ausstellungsräume}

Der damalige Museumsdirektor Rudolf Wegeli war sich bewusst, dass nur mit einem Erweiterungsbau die für die Orientalische Sammlung nötige Ausstellungsfläche geschaffen werden konnte. ${ }^{9} 1914$ wird deshalb der Architekt René von Wurstemberger (1857-1935) mit der Projekterarbeitung für einen Annexbau beauftragt. ${ }^{10}$ Nach Ausbruch des Ersten Weltkriegs gerät das Bauvorhaben zwar ins Stocken, man versucht aber dennoch, die Planung voranzutreiben. ${ }^{11}$ Moser beharrt darauf, dass die Sammlung in zusammenhängenden Räumen gezeigt werden soll, die direkt vom Haupteingang erreicht werden können und ausserdem mit einem Vestibül und einem tiefer liegenden Oberlichtsaal verbunden werden sollen. ${ }^{12}$ Ende 1916 kündigt Moser seine Absicht an, die Sammlung ,in einen orientalischen Rahmen zu bringen“. ${ }^{13}$ Er erwähnt Elemente der Innendekoration - etwa einen Fries und „Stalaktiten“ - und betont, dass ein definitives

8 Im Moser-Archiv im BHM ist umfangreiches Quellenmaterial (Korrespondenz, Protokolle, Pläne und Skizzen) vorhanden. Für den Aufsatz wurde teilweise nicht inventarisiertes Material verwendet, dieses ist mit dem Vermerk (BHM) gekennzeichnet. Die Autorin dankt Alban von Stockhausen, dem Kurator der ethnografischen Sammlungen des BHM, ausdrücklich für die Unterstützung der Recherchen.

9 Das 1894 eröffnete BHM hatte einen unerwartet grossen Sammlungszuwachs erlebt, und eine bauliche Erweiterung wurde bereits seit Ende des 19. Jhs. angestrebt. 1914 geriet das Museum durch die Schenkungen der Orientalischen Sammlung sowie eines Neuenburger StadtpalaisInterieurs aus dem 18. Jh. (Pourtalès-Salon) in Zugzwang; siehe Bernisches Historisches Museum 1918, 1-2.

$10 \mathrm{Zu}$ Wurstemberger siehe Schnell 2009.

11 Zur Baugeschichte des Annexbaus siehe auch Balsiger Kläy 1992, 190-197.

12 Siehe Protokoll Besprechung zwischen Moser, Wegeli und Lohner (Regierungsrat), 7.12. 1916 (BHM, Inv. 1916.670.181); Brief Moser an Wegeli, 25.4. 1917 (BHM, Inv. 1917.670.186).

13 Moser an Wegeli, 26.12.1916 (BHM, Inv. 1916.670.184). 
Projekt erst ausgearbeitet werden könne, „nachdem ich dasselbe mit meinem Pariser Architekten vereinbart habe“. ${ }^{14}$

Es ist der französische Architekt Henri Saladin (1851-1923), mit dem Moser ein Installationsprojekt für die Sammlung ausarbeiten will. In Bern begrüsst man seinen Vorschlag: „,[Wir] schätzen uns glücklich, dass Sie eine so bewährte Kraft in den Dienst ihrer Sache stellen wollen “. ${ }^{15}$ Saladin galt als Spezialist für islamische Baukunst, er hatte dazu mehrere Studien publiziert und mit dem ersten Band des Manuel d'art musulman: L'architecture (Paris, 1907) ein wichtiges Referenzwerk geschaffen. ${ }^{16}$ Für Moser hatte Saladin bereits die Ausstellungsinstallation und den Fumoir arabe auf Charlottenfels konzipiert. ${ }^{17}$

Anfang 1917 schickt Moser das bereits vorhandene Planmaterial nach Paris, um den Architekten über den Projektstand in Kenntnis zu setzen. ${ }^{18}$ Saladin bereitet sich seinerseits vor, indem er die Sammlung Pauilhac in Paris besichtigt. Der Industrielle Georges Pauilhac (1871-1958) hatte sich an seinen Wohnsitz (Avenue Malakoff 59) einen Ausstellungsraum für seine umfangreiche Waffenund Rüstungssammlung anbauen lassen, der ähnliche Gestaltungsprinzipien aufweist wie der geplante Berner Ausstellungsraum. So führen einige Stufen abwärts in einen von Oberlichtern beleuchteten Saal. ${ }^{19}$ Saladin wird vom Notar Charles Buttin begleitet, einem bekannten Waffenhistoriker und Sammler orientalischer Waffen. Wie Saladin an Moser schreibt, äusserte Buttin bei dem Treffen eine Idee für die Gestaltung des Waffensaals in Bern: „[I]l serait d'avis de disposer autour de la salle des armes une série d'arcades peintes de peu de profondeur [...], c'est une excellente idée“. ${ }^{20}$ Im Juni 1917 entwerfen Saladin und Moser gemeinsam die Grundkonzeption des Projekts. ${ }^{21}$ Bevor der Architekt 1918

14 Moser an Wegeli, 26.12.1916 (BHM, Inv. 1916.670.184).

15 Wegeli an Moser, 3.4.1917 (BHM, Inv. 1917.670.186).

$16 \mathrm{Zu}$ Saladins Person ist wenig bekannt. Er schloss 1881 das Architekturstudium an der École des Beaux-Arts in Paris ab. Für islamische Kunst und Architektur begann er sich nach einer mehrmonatigen archäologischen Mission in Tunesien (1882-1883) zu interessieren, siehe Bacha 2011. Dem Briefkopf zufolge, den Saladin in der Korrespondenz mit Moser verwendet hatte, war er zu dieser Zeit auch „Architecte du Gouvernement“ und „Expert près le Tribunal Civile de la Seine“.

17 Womöglich war Moser durch Saladins Tunesische Pavillons an den Weltausstellungen von 1889 und 1900 in Paris auf den Architekten aufmerksam geworden, siehe Giese 2016, 154; Balsiger/Kläy 1992, 185. Zum Fumoir arabe (1907-1909) siehe Giese 2016; Giese/Varela Braga 2019.

18 Siehe Brief Moser an Wegeli, 25.4.1917 (BHM, Inv. 1917.670.186).

19 Siehe <http://parismuseescollections.paris.fr/en/node/96106\#infos-principales>, hier allerdings fälschlich als „Galerie d'armes de Monsieur Panilhac“ bezeichnet.

20 Saladin an Moser, 14. 5.1917 (BHM).

21 Siehe Grundriss der Ausstellungsräume von Saladin, Charlottenfels 20.6.1917 (BHM). 
einen detaillierten Vorschlag für die Gestaltung der Sammlungsräume und die Sammlungsaufstellung ausarbeitet, wird die Aufgabenteilung zwischen Saladin und Wurstemberger geklärt, der für die Ausführung vor Ort zuständig ist. Auch Saladins Honorar wird festgelegt. ${ }^{22}$ Nach Kriegsende hofft Moser, um nicht mit Bern brechen $\mathrm{zu}$ müssen, auf die rasche Bewilligung des Baukredits. ${ }^{23}$ Im Juli 1919 wird der Grundstein für den Anbau gelegt. ${ }^{24}$

\section{„Wie ein Märchen“ - Die Eröffnung der Ausstellung und des Waffensaals}

Am 21. Mai 1922 kann das Musée Moser in Bern im Beisein des zu dieser Zeit 78-jährigen Stifters eröffnet werden. ${ }^{25}$ Während die Fassade des neuen Annexbaus dem historisierenden Stil des Hauptbaus des BHM angepasst wurde, zeigt sich im Innern des obersten Stockwerks, in dem die Moser-Sammlung untergebracht ist, ein anderes Bild: ${ }^{26}$ „Wie ein Märchen muten den Besucher die orientalisch ausgestatteten Räume an“, vermeldet etwa die Thurgauer Zeitung. ${ }^{27}$ Wie bereits weiter oben gesagt, umfasste die Sammlung damals fünf Räume (Abb. 3). Vom ersten Treppenpodest des Haupttreppenhauses aus gelangte man in ein beinahe quadratisches Atrium, an das sich seitlich das Arabische Rauchzimmer, die Moser-Bibliothek und ein Ausstellungsraum für Textilien anschlossen. Geradeaus führte eine breite Treppe unter einem Spitzbogen in den tiefergelegenen grossen Waffensaal (Abb.1). Von den fünf Räumen sind heute nur

22 Bis dahin hatte Moser Saladin bezahlt, nun aber sollte der Architekt eine Pauschalsumme von 15'000 Franken aus dem Stiftungskapital erhalten. Dieses umfasste einen Baufonds von 50’000 Franken, welchen Moser für die Kosten von Innendekoration und Sammlungsinstallation zur Verfügung gestellt hatte. Siehe Stiftungsurkunde vom 26.1.1914 (BHM, Inv. 1915.670.0159); Briefe Moser an Wegeli, 26.12.1916 (BHM, Inv. 1916.670.184); 12.12.1917 (BHM, Inv. 1917.670.190); Brief Wegeli an Moser, 11.12.1917 (BHM, Inv. 1917.670.190).

23 Siehe Brief Moser an Saladin, 6.3.1919 (BHM). Die Kosten für den Bau sollten sich Kanton, Stadt und Burgergemeinde Bern teilen.

24 Siehe Balsiger/Kläy 1992, 195. Akten zur Baueingabe und -bewilligung von 1919 befinden sich im Stadtarchiv Bern (SAB_BB_1190).

25 Für die Neueröffnung 1922 wurden sämtliche Ausstellungen im BHM neu konzipiert, womit ehemals „kunterbuntes Vielerlei und Modergeruch“ verschwanden, siehe Die Berner Woche in Wort und Bild, 14, 1924.

26 Projekt Museumsgebäude BHM: André Lambert \& Stahl (Stuttgart) 1891; Ausführung: Eduard von Rodt 1892-1894, siehe Biland 1994.

27 Thurgauer Zeitung, 18.5.1922. 


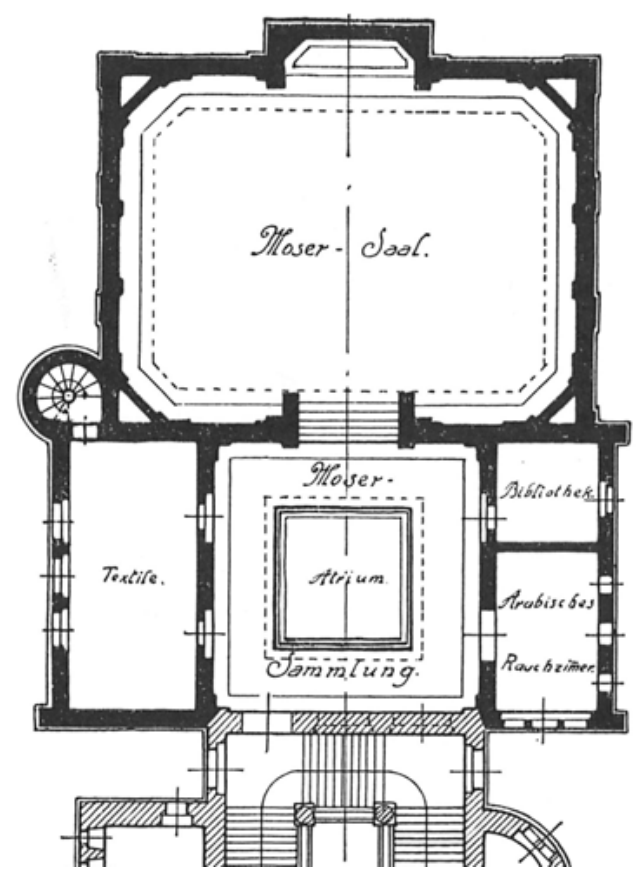

Abb. 3: Bernisches Historisches Museum, Grundriss Erweiterungsprojekt 1918 (Ausschnitt), Erweiterungsprojekt 1918, 1918, Tafel II. Bernisches Historisches Museum, Bern.

der Fumoir arabe (den man von Charlottenfels nach Bern gebracht hatte) und der Waffensaal in ihrer ursprünglichen Form erhalten (Abb. 4). ${ }^{28}$

Der grosse Oberlichtsaal im neo-timuridischen Stil bildete das Herzstück der Ausstellungsräumlichkeiten und stand während der Planung des Musée Moser stets im Mittelpunkt. ${ }^{29}$ Hier wurden die Waffen - sie bilden den Kern der Sammlung -, sowie Rüstungen und Bronzearbeiten gezeigt. Heute wird nur

28 Die Orientalische Sammlung und die dafür konzipierten Räumlichkeiten erlebten ab 1969, als die Moser-Ausstellung nach knapp 50 Jahren geschlossen wurde, ein wechselvolles Schicksal. Der Saal diente zunächst als Lagerraum, und die kleineren Sammlungsräume wurden (nach der Entfernung des Dekors) für andere Ausstellungszwecke eingesetzt. 1989 wurde eine neu konzipierte Ausstellung zur Orientalischen Sammlung eröffnet. 2007 fand eine weitere Umgestaltung statt, mit der Folge, dass heute nur noch ein kleiner Teil der Moser-Sammlung im grossen Saal gezeigt wird; siehe Balsiger/Kläy 1992, 197-202; Kläy 1994, 349-356.

29 Saladin stellte Anfang 1919 sämtliche Pläne und Detailzeichnungen fertig, die man in Bern zur Umsetzung des Interieurs benötigte. Der Architekt liess Modelle des Frieses im Massstab 1:1 herstellen, die abgeformt und nachgegossen werden konnten; siehe Briefe Saladin an Moser, 22.1.1919; 18.3.1919 (BHM). 


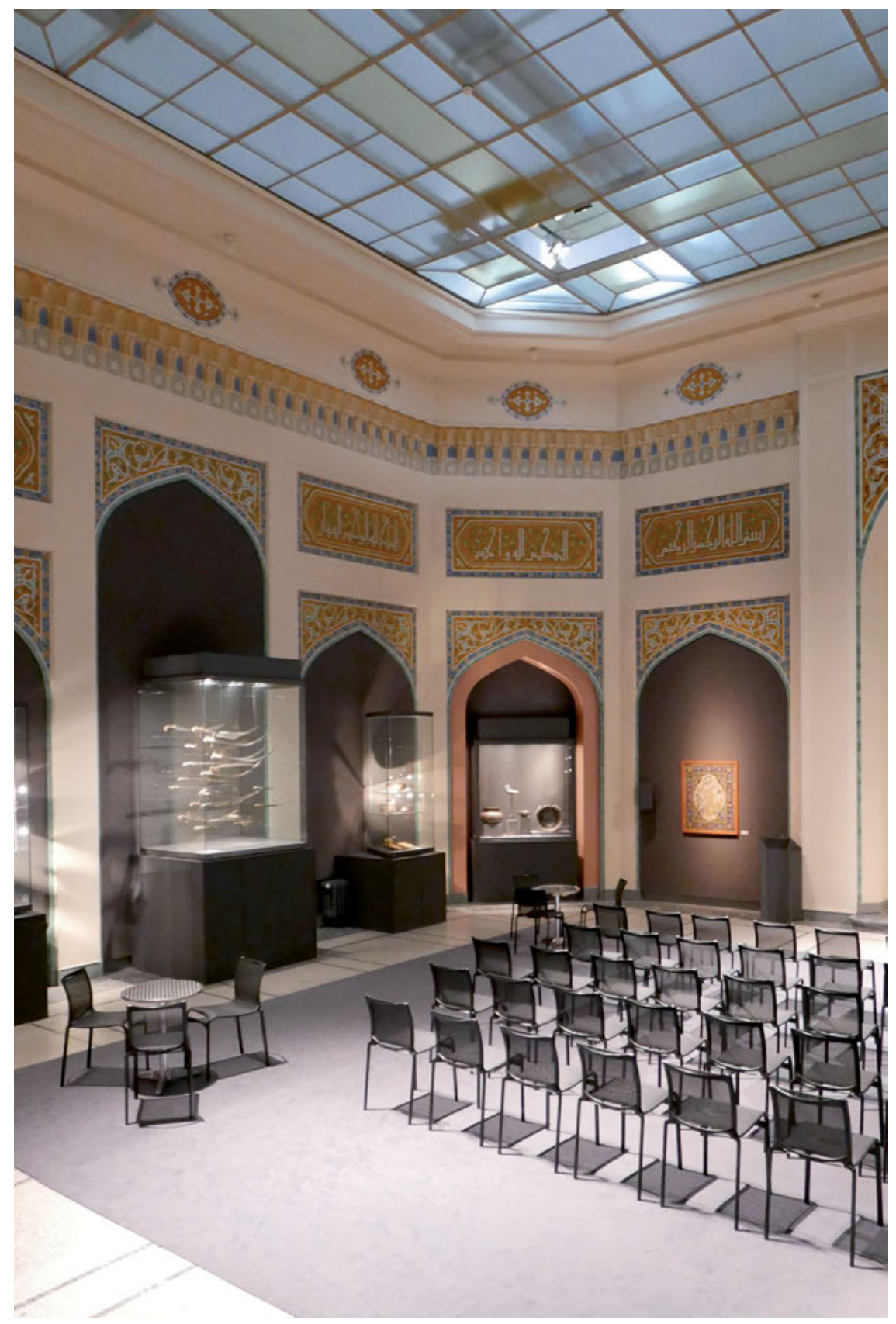

Abb. 4: Bern, Bernisches Historisches Museum, Moser-Saal, Henri Saladin, 1917-1922. Fotografie Katrin Kaufmann, 2016. 
noch ein kleiner Teil der Moser-Sammlung in Vitrinen entlang der Wände ausgestellt. Der 13.5×18 m messende Raum wird hauptsächlich als Veranstaltungsort genutzt, hat aber trotz der Umnutzung weitgehend sein Aussehen behalten. ${ }^{30}$

Die Wände des $8.5 \mathrm{~m}$ hohen Raumes sind mit spitzbogigen Arkaden gegliedert, die leicht vertiefte Nischen rahmen. Die Ecken des Saals wurden mit Schrägwänden kaschiert. Gegenüber dem Eingang liegt eine Nische, die von einem Backsteingewölbe mit weissen Rippen überwölbt ist. Im oberen Teil der Wände verläuft ein Fries mit prismatischen Elementen. Die architektonische Gestaltung wird durch einen nicht weniger wichtigen malerischen Dekor ergänzt. Die Bereiche zwischen den Arkadenbogen und ihrer orthogonalen Umfassung sind mit Rankenwerk verziert. Rechteckige Schriftfelder und ein ovales, in regelmässigen Abständen über dem Fries appliziertes Motiv komplettieren den Dekor.

\section{Konzeption des Waffensaals - Vorbilder, Vorlagen, Variationen}

Moser hatte nicht nur konkrete Vorstellungen davon, wie der Waffensaal angelegt werden sollte (Oberlicht und Niveau-Unterschied zum Atrium), er bestimmte auch ein Vorbild für das Dekor: das Gur-i Amir in Samarkand, das Mausoleum Timur Lenks (gest. 1405), Begründer der Dynastie der Timuriden. Mosers Architekt Saladin galt zwar als ausgewiesener Kenner islamischer Architektur und hatte mehrere Reisen, etwa nach Nordafrika, unternommen, nie jedoch nach Zentralasien. ${ }^{31}$ Für die Gestaltung des Ausstellungssaales war der Architekt deshalb auf Vorlagematerial angewiesen. Bereits für den Manuel d'art musulman musste Saladin auf Literatur und Bildmaterial zurückgreifen. So etwa im Kapitel zur École Persane, in dem er auch die stark von der persischen Architektur abgeleitete Baukunst der Timuriden abhandelte. Saladin hatte damals unter anderem Fotografien von Paul Nadar (1856-1939), aber auch von Moser erhalten. Mosers Fotografie des Gur-i Amir wurde in Saladins Buch abgedruckt (Abb. 2). ${ }^{32}$ Für die Planung des Ausstellungsraums griff der Architekt wieder auf diese Bildquellen zurück: „J'ai mis de côté des grandes vues de Nadar sur les mos-

30 Die grosse Bogenöffnung zwischen Atrium und Saal wurde verschlossen, der Zugang zum Saal erfolgt heute über einen Durchgang in der Trennwand.

31 Laut Saladins Angaben im Manuel d'art musulman hatte er bis 1907 die Türkei, Ägypten, Palästina, Syrien, Tunesien und Algerien bereist. Siehe Saladin 1907, XI.

32 Siehe Saladin 1907, 363; Briefe Saladins an Moser, 10. 5. 1905 (BHM, Inv. 1922.670.0260.81); 21. 9.1905 (BHM, Inv. 1922.670.0260.81). 
quées de Samarcande ainsi que votre collection si intéressante que vous m’avez donnée autrefois“. 33

Wie aus Saladins Briefen und seinem Projektbeschrieb von 1918 deutlich wird, sollte der Ausstellungsraum durch die Wandgliederung mittels Arkaden an den Innenhof einer madrasa - einer islamischen Lehrstätte für Theologie und Recht - erinnern. ${ }^{34}$ Ähnlich wie beispielsweise bei den Medresen am Registan-Platz in Samarkand, die um arkadengesäumte Innenhöfe nach dem VierIwan-Schema (bestehend aus vier kreuzförmig angeordneten, zum Hof offenen Hallen) angelegt waren, war im Berner Ausstellungsraum auf allen vier Seiten jeweils die zentrale Arkade akzentuiert. Die Dimensionierung der beiden grössten Arkadenbogen des Waffensaals entwickelte Saladin anhand von im Manuel d'art musulman gezeigten Beispielen. ${ }^{35}$

Die grosse überwölbte Nische bezeichnete Saladin, den Gebetsnischen in Moscheen entsprechend, als mihrab. Der Architekt schlug erst einen rechteckigen Grundriss vor, in Analogie zu den Nischen im Gur-i Amir, später jedoch einen trapezförmigen Grundriss, der sich besser für das Ausstellen von Waffen zu eignen schien. ${ }^{36}$ Für die Architektur Samarkands wäre ein muqarnas-Gewölbe typisch gewesen - Saladin entschied sich aus Kostengründen jedoch für eine „voûte à nervures persanes““. ${ }^{37}$ Die Farbe der Backsteine im Gewölbe sollte sehr blass sein, „comme la terre de Samarcand dont j'ai un échantillon entre les mains“. 38

Als Vorlage für die Formgebung und die Bemalung des Frieses nutzte Saladin eine Fotografie Nadars, welche die muqarnas-Nische über dem Portal eines timuridischen Mausoleums in der Nekropole Shah-i Zinda von Samarkand zeigt. ${ }^{39}$ Das nach Bern übertragene Element ist in Samarkand im unteren Teil der Nische zu finden, wo es zum dreidimensionalen muqarnas-Gewölbe überleitet. In Aneinanderreihung erfüllt es in Bern eine rein dekorative Aufgabe (Abb. 5).

33 Saladin an Moser, 16.7.1917 (BHM).

34 Siehe Brief Saladin an Moser, 28.3.1918 und Projektbeschrieb Saladin, 5. 5.1918, 2 (BHM).

35 Die Dimensionierung der beiden grössten Arkadenbogen des Waffensaals entwickelte Saladin anhand von im Manuel d'art musulman gezeigten Beispielen, so etwa der Sher-Dor-Madra$s a$ in Samarkand und der Freitagsmoschee in Isfahan, siehe Saladin 1907, 328/363; Brief Saladin an Moser, 8.4.1918 (BHM).

36 Siehe Briefe Saladin an Moser, 9.1.1918; 19.1.1918 (BHM).

37 Saladin an Moser, 19.1.1918 (BHM).

38 Saladin an Moser, 15.4.1918 (BHM).

39 Siehe Briefe Saladin an Moser, 28. 3. 1918; 31. 5. 1918; 3.6. 1918 (BHM) und Saladin 1907, 357. Nadars Fotografie zeigt ein Detail des ab 1371 errichteten Grabmals Shad-i Mulk Aqa, Saladin spricht hingegen vom „Tombeau de Tschouchouk Bika“; siehe Brief Saladin an Moser, 28.3.1918 (BHM). 


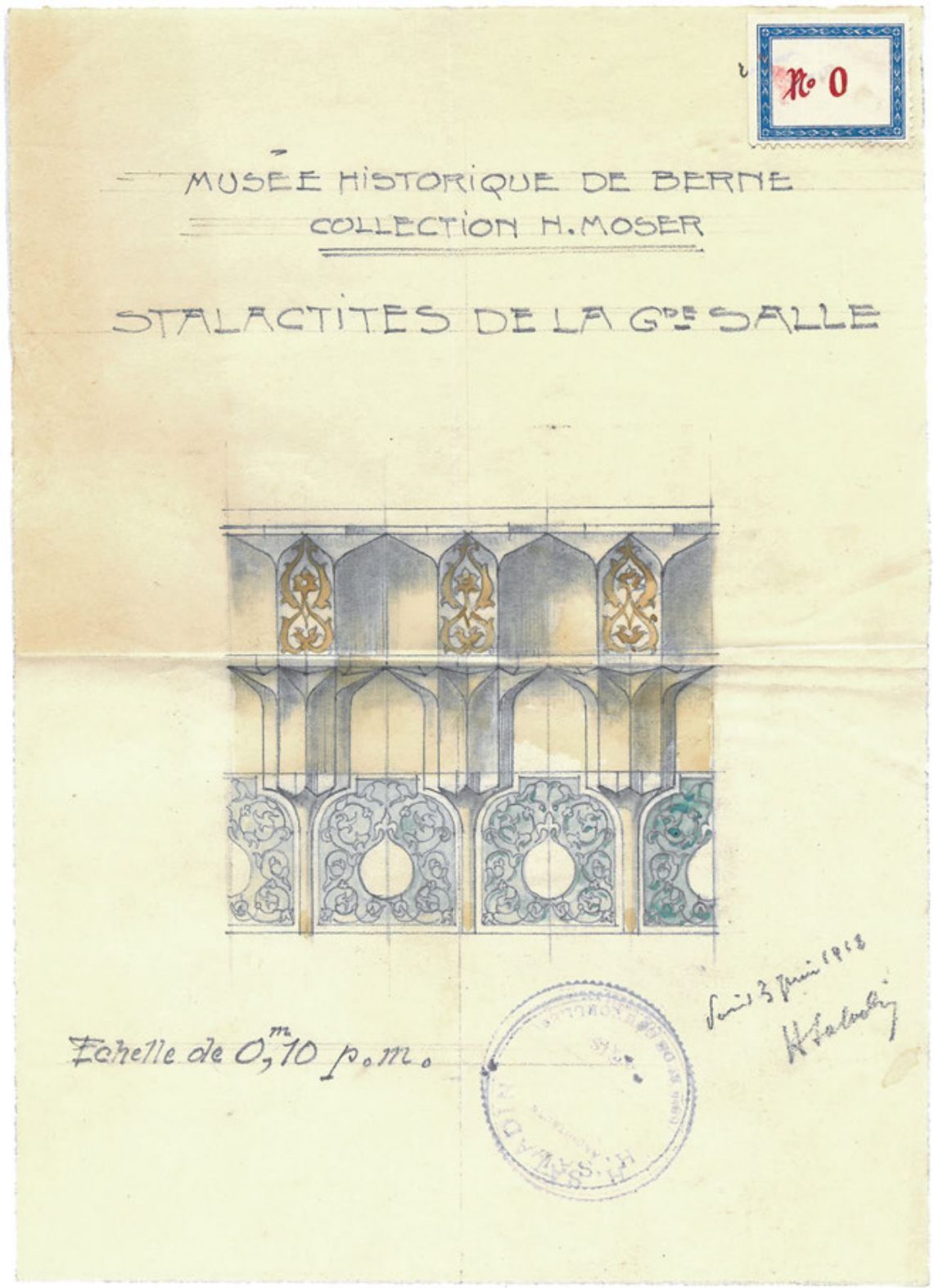

Abb. 5: Henri Saladin, Collection H. Moser, Stalactites de la grande salle, 3. 6.1918. Papier, Bleistift/Aquarell, $162 \times 224 \mathrm{~mm}$. Bernisches Historisches Museum, Bern. 


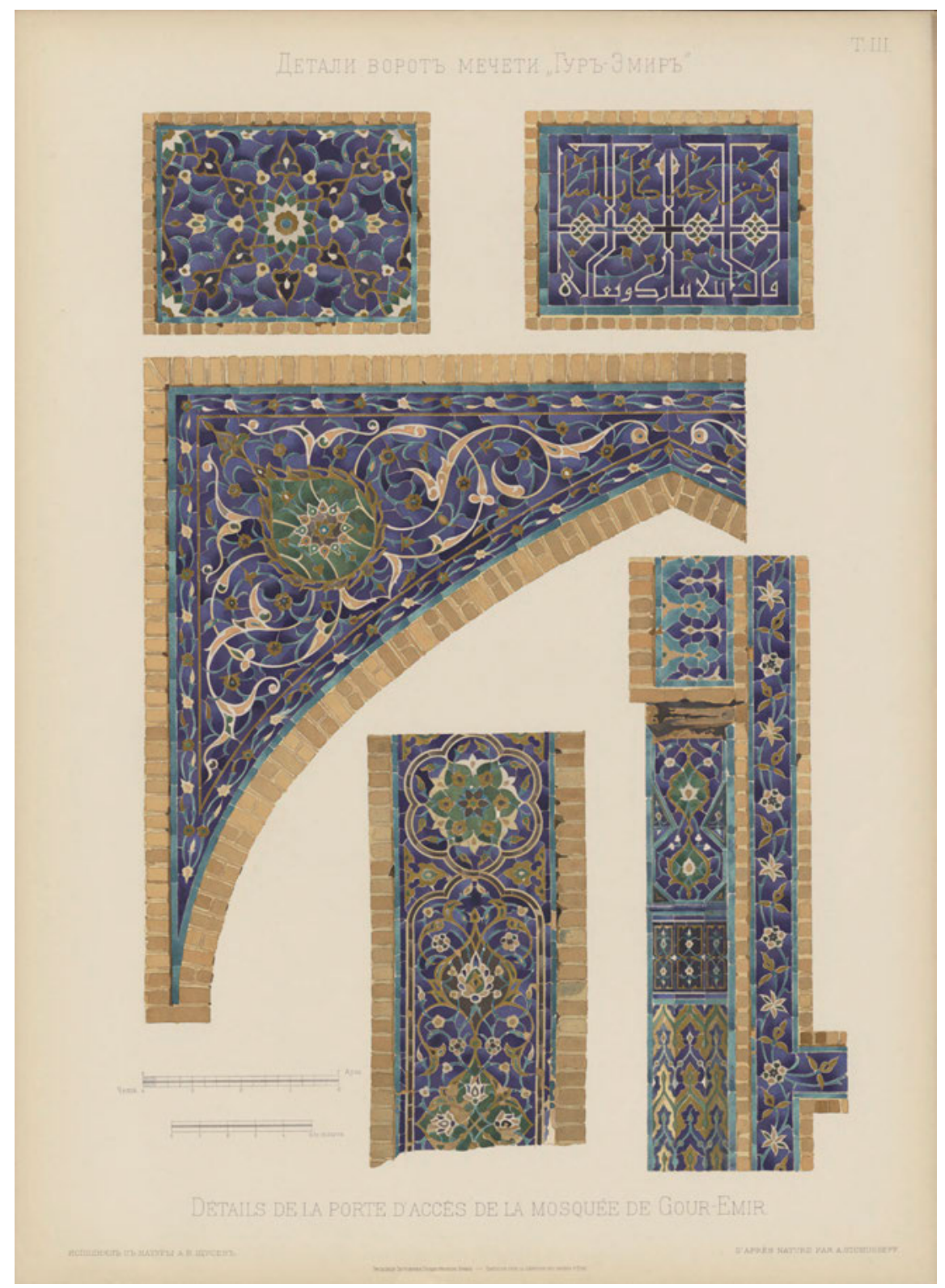

Abb. 6: Commission Impériale Archéologique, Détails de la porte d'accès de la mosque de Gour Emir, Les Mosquées de Samarcande: Fascicule I, Gour-Emir, 1905, Tafel III. The Cleveland Museum of Art. 
Der malerische Dekor, in dem orange und türkisblaue Farbtöne neben Weiss und Grün dominieren, imitiert die Verkleidung mit Fayence-Mosaiken, die für viele Gebäude in Samarkand - so auch für das zu Beginn des 15. Jahrhunderts erbaute Gur-i Amir - charakteristisch ist. ${ }^{40}$ Das Motiv mit Rankenwerk, welches die Berner mihrab-Nische im oberen Teil umfasst, ist eindeutig vom monumentalen Eingangsportal (pištaq) der madrasa übernommen, an die das Mausoleum in Samarkand angegliedert war, variiert jedoch in der Farbgebung. ${ }^{41}$ Saladin versicherte Moser mehrfach, er habe sich auf die Farbtafeln der Publikation Les Mosquées de Samarcande: Gour-Emir (Sankt Petersburg, 1905) bezogen, die ihm Moser ausgeliehen habe (Abb. 6). ${ }^{42}$ Für das ovale Motiv oberhalb des Frieses hat ihm hingegen wohl ein - hier stark vereinfachtes - oft in der persischen Buchmalerei verwendetes Element als Vorbild gedient. ${ }^{43}$

Die Ausführungen zeigen, dass Saladin sich einzig für die malerische Ausstattung des Saales tatsächlich am Gur-i Amir orientiert hatte. Für die architektonische Ausgestaltung kombinierte er stilisierte Elemente timuridischer und persischer Baukunst miteinander und passte sie den Bedürfnissen der Ausstellungsinstallation an.

\section{Eine Konzession an die Museumsleitung - Koransprüche statt zentralasiatischer Landschaften}

Das Projekt für die Ausstellungsräumlichkeiten wurde vorwiegend zwischen Saladin und Moser besprochen. Es sind nur wenige Quellen überliefert, die Aufschluss darüber geben, wie die Museumsleitung auf die Ausgestaltung der Räume Einfluss nahm. In der Broschüre zum Erweiterungsprojekt gab man sich zuversichtlich:

Jedenfalls bürgt uns der Name des Herrn Dr. Moser, der im Ausstellungswesen eine Autorität ist, in Verbindung mit seinem Mitarbeiter Saladin dafür, dass die Sammlung nicht

40 Die verwendeten lackähnlichen Farben sollten den Glanzeffekt von Fayence imitieren. Bei der Restaurierung des Dekors 1985/86 wurden die Farben offenbar leicht abgetönt; wie sehr sie sich heute von der ursprünglichen Version unterscheiden, wäre noch zu untersuchen.

41 Siehe Brief Saladin an Moser, 1.5.1918 und Projektbeschrieb Saladin, 5. 5.1918, 9 (BHM).

42 Siehe Commission Impériale Archéologique 1905 und Brief Saladin an Moser, 28.2. 1918 (BHM).

43 In der Sammlung befindet sich ein persisches Manuskript, auf dessen Bucheinband ein ähnliches Motiv in Leder aufgebracht ist, siehe Zeller 1922, 204. Moser hatte ein vergleichbares Motiv für den Umschlag seines Waffenkatalogs verwendet, siehe Moser 1912. 


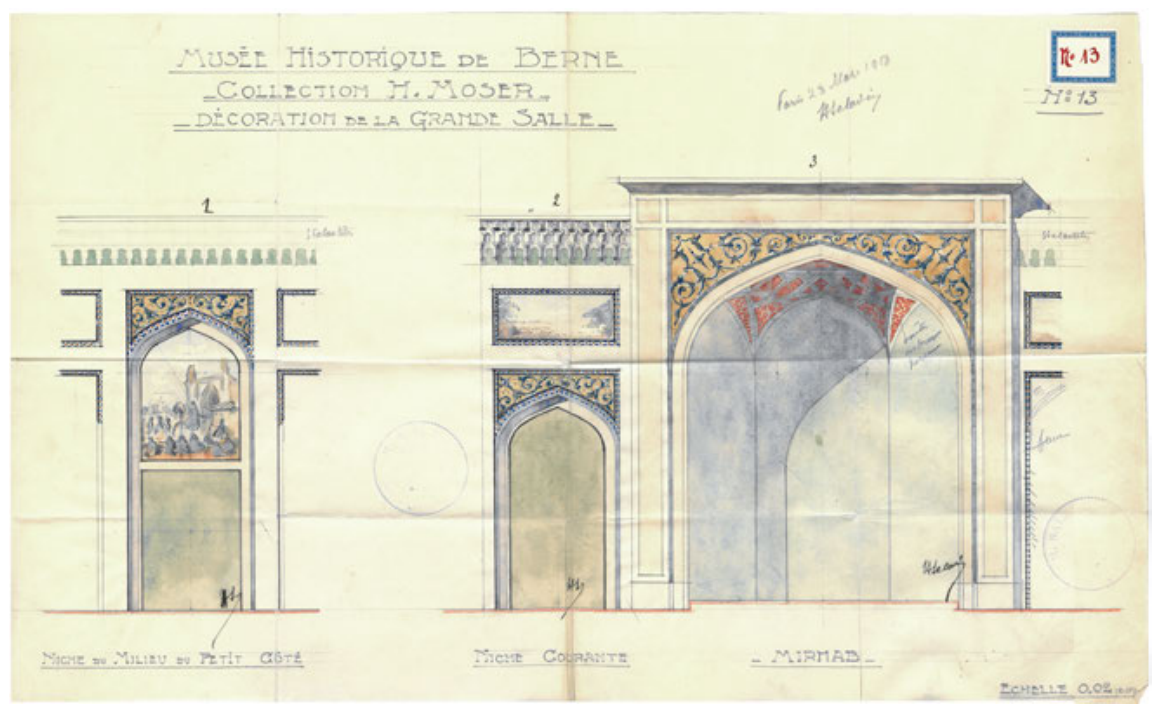

Abb. 7: Henri Saladin, Collection H. Moser, Décoration de la grande salle, 28. 3.1918. Papier, Bleistift/Aquarell, $454 \times 270 \mathrm{~mm}$. Bernisches Historisches Museum, Bern.

nur in Bezug auf ihren Inhalt, sondern auch in der Art ihrer Installation, eine Sehenswürdigkeit darstellen wird. ${ }^{44}$

Eine Aussage Saladins gegenüber Moser legt nahe, dass in Bern eine zurückhaltende Gestaltung gewünscht war. ${ }^{45}$

Die Aufsichtskommission der Stiftung schaltete sich ein, als es um eine Serie von Malereien ging, die im Waffensaal auf die Wand appliziert werden sollte. Das von Saladin erarbeitete Projekt sah vor, den ornamentalen Dekor des Saales durch gegenständliche Malereien in den mittleren Nischen der Querwände und in rechteckigen Wandfeldern über den Bogenarkaden $\mathrm{zu}$ ergänzen (Abb. 7). Moser ging nach einem ihm vertrauten Prinzip vor: Hatte er früher seine Ausstellungen einzig mithilfe gewaltiger Draperien aus orientalischen Textilien in Szene gesetzt, so waren mit den Bosnischen Pavillons an den Weltausstellungen in Brüssele (1897) und Paris (1900) Malereien hinzugekommen, die Landschaften und Städte zeigten. ${ }^{46}$ Moser wollte in Bern zentralasiatische

44 BHM 1918, 7.

45 „D’abord je vous remercie de ce que vous me dites de mon projet qui devrait, d'après certains rester presque anonyme, et de ce que vous prendrez ma défense“, Brief Saladin an Moser, 19.-22. 2. 1918 (BHM).

46 Moser war verschiedentlich als Ausstellungsmacher tätig gewesen. Bevor er seine Sammlung auf Charlottenfels installierte, hatte er sie hauptsächlich ab 1886 in verschiedenen 
Landschaften und wenige Stadtansichten mit figurativen Alltagsszenen zeigen, für die der Pariser Maler Georges Bertin Scott (1873-1943) Bildvorlagen erstellen sollte. ${ }^{47}$ Scott war unter anderem bereits an der Gestaltung des Folio-Prachtbands Sammlung Henri Moser-Charlottenfels. Orientalische Waffen und Rüstungen (Leipzig, 1912) beteiligt gewesen und hatte damals anhand von Fotografien Mosers eine Skizze des Registan-Platzes in Samarkand gemalt. ${ }^{48}$ Moser beabsichtigte, die definitiven Sujets für den Waffensaal zusammen mit Scott auf Charlottenfels zu bestimmen. Der Künstler sollte sie dann anhand von Fotografien, Objekten aus der Sammlung sowie der Zeichnungen Evert van Muydens (1853-1922) aus Mosers Reisebericht À travers l'Asie centrale (Paris, 1885) umsetzen. ${ }^{49}$ Die Malereien waren fast zwei Jahre lang ein Thema in der Korrespondenz zwischen Moser, Saladin und Scott. Bevor es jedoch zu dem Treffen auf Charlottenfels kam, entschied sich die Aufsichtskommission der Stiftung gegen die Gemälde. ${ }^{50}$ Rudolf Zeller, Kurator der Ethnografischen Abteilung und Sekretär der Aufsichtskommission des BHM, dürfte hierbei eine ausschlaggebende Rolle gespielt haben. Zeller hatte gegenüber Moser bereits früher Bedenken geäussert und vorgeschlagen, statt der Malereien Koransprüche anzubringen. ${ }^{51}$ Damit war wiederum Moser nicht einverstanden: Von „Koranischen Sprüchen und Sentenzen“ sei abzusehen, denn diese hätten nur dann einen Wert, wenn sie durch berühmte Kalligrafen aufgesetzt würden, was aber zu teuer wäre. ${ }^{52}$

Nach dem Entscheid der Aufsichtskommission schlug auch Saladin vor, anstelle der Landschaften dekorative Schriften zu verwenden. Um dem Publikum die Herkunft der Moser-Sammlung zu vermitteln, sollten auf Persisch oder Arabisch - besser noch auf Französisch in dekorativen gotischen Lettern - die Namen der Städte und Länder angebracht werden, aus denen die Sammlungs-

Schweizer Städten sowie in Stuttgart und Paris gezeigt. Als Generalkommissär für BosnienHerzegowina war er für die Konzeption der bosnischen Pavillons an den Weltausstellungen in Brüssel (1897) und Paris (1900) verantwortlich, siehe Balsiger/Kläy 1992, 167-190.

47 Saladin hatte erst die Reproduktion persischer Miniaturen vorgeschlagen, siehe Briefe Saladin an Moser, 19. 8.1917; 19.1.1918; 10.5.1919; und Brief Moser an Saladin, 22. 2.1918 (BHM). 48 Scott hatte die Erstellung der Bildtafeln begleitet, zudem sind dem Katalog drei seiner Zeichnungen vorangestellt. Die 1912 verfertigte Gouache des Registan-Platzes (abgedruckt in Balsiger/Kläy 1992, 132) war als Vorlage für die Gestaltung einer der zentralen Nischen an den Querwänden vorgesehen. Für die gegenüberliegende Nische zog Saladin eine Darstellung der Nekropole Shah-i-Zinda in Samarkand in Betracht. Siehe Brief Saladin an Moser, 19.1.1918; Brief Moser an Scott, 27. 2.1919 (BHM).

49 Siehe Briefe Moser an Saladin, 22. 2. 1918; 20.3.1918; Moser an Scott, 27. 2.1919 (BHM).

50 Siehe Brief Saladin an Moser, 10.5.1919 (BHM).

51 Siehe Brief Moser an Zeller, 7.11.1918 (BHM).

52 Siehe Brief Moser an Zeller, 7.11.1918 (BHM). 
objekte stammten. ${ }^{53}$ Zur Umsetzung kam jedoch Zellers Vorschlag: Zwölf rechteckige Wandfelder wurden mit insgesamt sechs unterschiedlichen Koransprüchen und Segenswünschen in einer stilisierten Form kufischer Schrift geschmückt. Die Inschriftenreihe beginnt links der mihrab-Nische mit der basmala, der Anrufungsformel: bi-smi Allāhi al-rahmāni al-rahīmi („Im Namen des barmherzigen und gnädigen Gottes“). Sie endet beim Bogen zum Atrium mit der Ermahnung 'amara Allāhu bi-'l-wifāqi wa-al-'adli (,,Gott befiehlt Eintracht und Gerechtigkeit“). Wahrscheinlich stammt die definitive Version der mit Ornamenten unterlegten Schriften von Stettler und Hunziker, den Architekten, die 1919 die Bauleitung für den Anbau übernahmen. ${ }^{54}$ Sie könnten Zellers Vorschlag aufgenommen haben, einen Koran aus der Moser-Sammlung als Vorlage zu nehmen oder den Spezialisten Samuel Flury (1874-1935) beizuziehen. ${ }^{55}$ Am BHM bevorzugte man also anstelle opulenter malerischer Darstellungen eine museale, wissenschaftlich-sachliche Inszenierung - die Aufsichtskommission hatte hier an entscheidender Stelle in den Gestaltungsprozess eingegriffen. ${ }^{56}$

\section{Entgegen einer Modeerscheinung - Samarkand und Abgrenzung vom Alhambra-Stil}

Es gibt mehrere Gründe, warum Moser für den gewünschten orientalischen Rahmen den neo-timuridischen Stil wählte. Betonen wollte er damit wohl die geographische Herkunft seiner Sammlung, die laut Eigenaussage aus „Waffen und Gegenständen der mohammedanischen Kunst und des Kunstgewerbes [...] namentlich aus Zentralasien und Persien“ bestand. ${ }^{57}$ Den Kern der Sammlung bil-

53 Siehe Briefe Saladin an Moser, 10.5.1919; 19.5.1919; 24. 5.1919 (BHM).

54 Siehe Brief Moser an Saladin, 27.2.1919 (BHM). Von Stettler und Hunziker liegt eine Entwurfszeichnung zu einem Schriftfeld vor (BHM).

55 Siehe einen ungenau datierten Brief Zellers an Moser von Dezember 1918 (BHM). Samuel Flury, ein Pionier auf dem Gebiet der islamischen Epigrafik und Kunst, hatte die Ornamentik des Kufi-Duktus erforscht. Seine bekannteste Studie erschien kurz vor der Umsetzung des Berner Ausstellungssaales, siehe Flury 1920.

56 Siehe Balsiger/Kläy 1992, 194. Der Fumoir arabe - eine Kompilation von Artefakten und zeitgenössischen Kopien - entsprach als Exponat wohl ebenfalls nicht den Vorstellungen der Museumsleitung. Als Moser aufgrund der Revolution in Russland viel Geld verliert und darauf angewiesen ist, einen Teil der Sammlung zu veräussern, schlägt Bern vor, den Fumoir zu verkaufen; siehe Brief Droin (Advokat) an Lohner (Regierungsrat), 10.8.1920 (BHM, Inv. 1920.670.106).

57 Stiftungsurkunde Orientalische Sammlung vom 26.1.1914 (BHM, Inv. 1915.670.0159). 
deten nämlich jene Objekte, die er von seinen insgesamt vier Reisen in Zentralasien mitgebracht hatte. ${ }^{58}$ Die Wahl des Gur-i Amir als Vorbild zeugt auch von Mosers Begeisterung für die Stadt Samarkand und ihr architektonische Erbe, die im eingangs angeführten Zitat aus À travers l'Asie centrale zum Ausdruck kommt. ${ }^{59}$

Aus einem Brief an Saladin geht zudem hervor, dass Moser sich dezidiert von einer Mode in der Interieur-Gestaltung abgrenzen wollte, die sich im 19. Jahrhundert in ganz Europa verbreitet hatte: „Je ne [sic] veux absolument exclure dans la décoration de la salle le style si vulgaire de l'Alhambra que l'on retrouve dans tous les Cafés concerts“. ${ }^{60}$ Die architektonische Rezeption der mittelalterlichen Nasridenpaläste in Granada hatte sich erst in Form neo-maurischer Interieurs an Herrscherhöfen und in Adelsresidenzen niedergeschlagen und wurde im Anschluss immer weiter popularisiert. Zur Zeit der Planungen in Bern hatte die Mode ihren Zenit längst überschritten. Wie Saladin beschwichtigend an Moser schreibt, entspreche sein Entwurf hingegen dessen Vorgaben: „Vous verrez que cela n'a rien de l'Alhambra, mais que c'est purement persan comme toute l'architecture du Turkestan“. ${ }^{61}$ Moser selbst betont gegenüber seinem Architekten, welchen Vorteil es habe, das Projekt am Gur-i Amir zu orientieren: „Cela nous fera quelque chose de tout à fait nouveau et surtout différent du style Alhambra que vous savez ne pas être de mon goût“. ${ }^{62}$

\section{„Ein Werk, das uns überlebt“}

Der Waffensaal im BHM ist zwar ein spätes neo-islamisches Interieur, vom Stil her aber tatsächlich etwas Neues und Einzigartiges. In Europa sind unzählige Beispiele architektonischer Orientalismen bekannt - die zentralasiatische Baukultur wurde jedoch kaum rezipiert. Aufgrund der seltenen Aneignung timuridi-

58 Mosers reiste zwischen 1868 und 1890 vier Mal nach Zentralasien. Sein Reisebericht $\grave{A}$ travers l'Asie centrale basiert auf der dritten Reise (1883-1884).

59 Siehe Moser 1886 [1885], 110, 112-119.

60 Moser an Saladin, 15.2.1918 (BHM). Der bei orientalisierenden Interieurs des 19. Jhs. oft angetroffene Stilmix war von Moser und Saladin ebenfalls nicht erwünscht. Überlegungen, die Nischen mit einem reliefierten Lincrusta-Wandbelag auszukleiden, wurden wieder verworfen, da das Material nur mit einem Dekor in maurischem Stil erhältlich war und die Herstellung des Materials nach eigenem Entwurf zu kostspielig gewesen wäre, siehe Brief Saladin an Moser, 6.11.1918 (BHM).

61 Saladin an Moser, 28.3.1918; siehe auch Brief Saladin an Moser, 28. 2. 1918 (BHM).

62 Moser an Saladin, 20.3.1918 (BHM). 
scher Dekorelemente ist der Saal ein bedeutsames architektonisches Denkmal. ${ }^{63}$ Des ungeachtet stand für Moser stets die Sammlung als solche im Vordergrund - die Gestaltung des Ausstellungsraumes sollte lediglich dazu beitragen, die Sammlungsobjekte wirkungsvoll zu präsentieren. ${ }^{64}$ Laut eigenen Angaben war die Installation der Sammlung im Museum für Moser „gewissermassen eine Lebensfrage“. ${ }^{65}$ Nach Mosers Schenkung vergingen mehr als acht Jahre, bis Wegeli dem Stifter endlich vermelden konnte: „Ihre Sammlung ist ausgestellt und kommt in dem schönen Rahmen wunderbar zur Geltung“. ${ }^{66}$ Diese Lebensfrage schien gelöst: Mosers Erbe, sein Lebenswerk, war gesichert.

Die Eingangstür des Musée Moser war mit einer Kartusche dekoriert, die eine Maxime des Persischen Dichters Saadi (13. Jahrhundert) wiedergab (Abb. 8): „Unser Ziel ist es, ein Werk zu schaffen, das uns überlebt“. ${ }^{67}$ Dass ausgerechnet ein Mausoleum als architektonische Quelle gewählt worden war, unterstreicht den Stellenwert, der dieser Sammlung für Moser zukam. Gleichzeitig weist dies auch darauf hin, dass hier nicht nur einer wissenschaftlichen Sammlung, sondern auch einer Person ein Denkmal geschaffen werden sollte.

Am 15. 7.1923, ein Jahr nach der Ausstellungseröffnung, verstarb Moser in Vevey. Obzwar heute nur ein Teil der Sammlung ausgestellt wird und die Ausstellungsräume nicht mehr in ihrer Gesamtheit erhalten sind, zeugt der Waffensaal im neo-timuridischen Stil noch immer von der faszinierenden Persönlichkeit Mosers - und von einem ebenso spannenden Kapitel der Schweizer Architekturgeschichte.

63 Zeitweise als Kitsch abgetan, wurde der Saal ab den 1980er Jahren als ,(innen-)architektonisches Dokument für den späten Historismus“ neu bewertet, Kläy 1994, 351.

64 Siehe Brief Moser an Saladin, 15.2.1918 (BHM).

65 Protokoll Besprechung zwischen Moser, Wurstemberger, Wegeli, Lohner (Regierungsrat) und Müller (Finanzdirektor), 6.11.1917 (BHM, Inv. 1917.670.185).

66 Wegeli an Moser, 1.5.1922 (BHM).

67 Moser hatte sie als Lebensmotto seinem Waffenkatalog von 1912 vorangestellt (siehe Moser 1912). Saladin hatte den Vorschlag gemacht, das Motto auch in Bern anzubringen; siehe Brief Saladin an Moser, 1.5.1918 (BHM). 


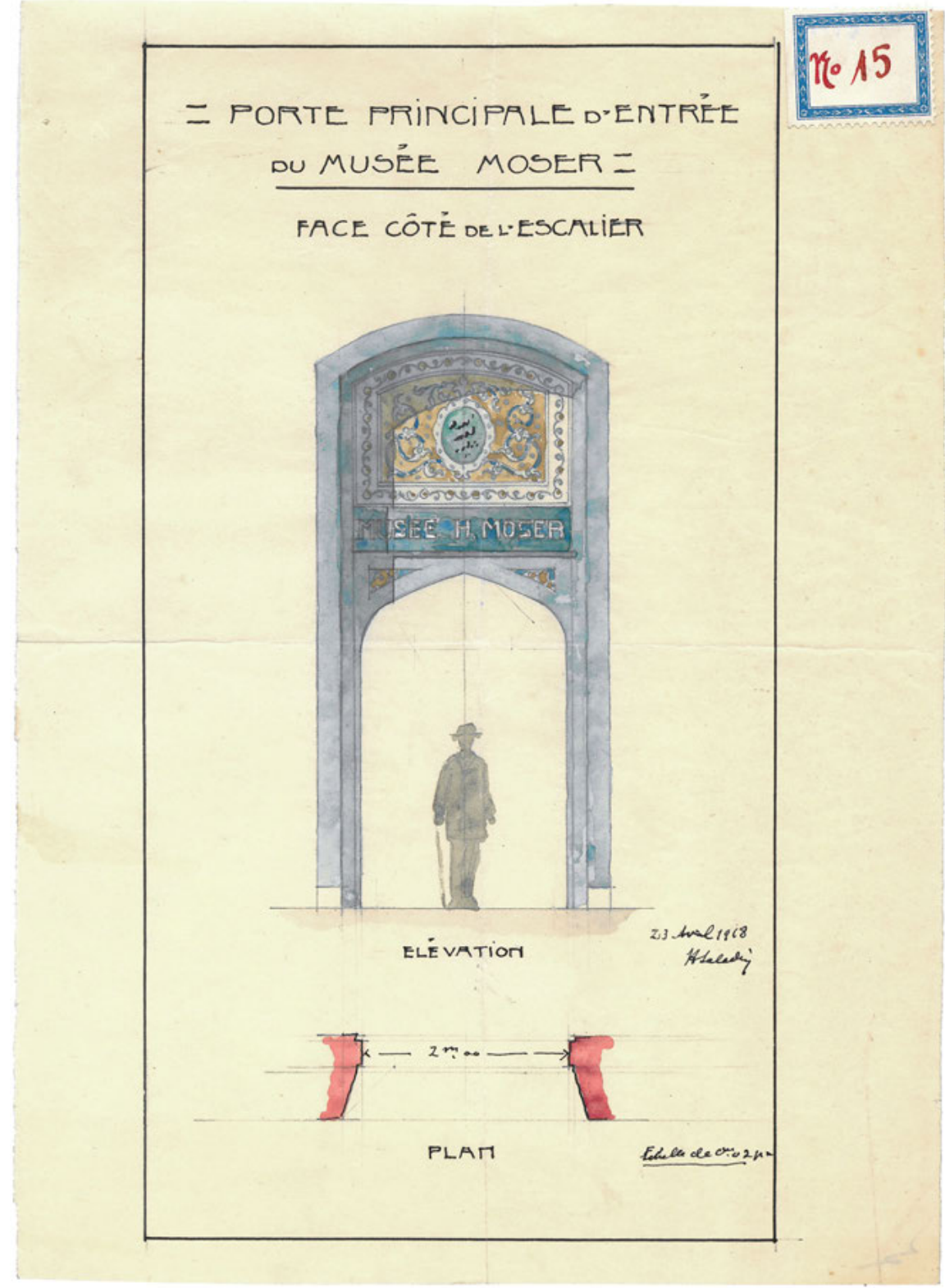

Abb. 8: Henri Saladin, Porte principale d'entrée du Musée Moser, 23. 4.1918. Papier, Bleistift/Aquarell, $180 \times 248$ mm. Bernisches Historisches Museum, Bern. 


\section{Bibliografie}

BACHA 2009, M. Bacha, „Henri Saladin (1851-1923). Un architecte ,Beaux-Arts“ promoteur de l'art islamique tunisien“, in N. Oulebsir und M. Volait (Hrsg.), L'Orientalisme architectural entre imaginaires et saviors, Paris, Picard (Collection d'une rive l'autre), 2009. <http://inha.revues.org/4916> (Seite abgerufen am 2. 2. 2017).

BALSIGER/KLÄY 1992, R. Balsiger und E. Kläy, Bei Schah, Emir und Khan: Henri Moser, Charlottenfels, 1844-1923, Schaffhausen, Meier, 1992.

Bernisches HIStorisches Museum 1918, Bernisches Historisches Museum, Erweiterungsprojekt 1918, Bern, Bernisches Historisches Museum, 1918.

BILAND 1994, A.-M. Biland, Bernisches Historisches Museum: Architekturführer, Bern, GSK (Schweizerische Kunstführer, 549), 1994.

Commission Impériale Archéologique 1905, Commission Impériale Archéologique, Les Mosquées de Samarcande: Fascicule I, Gour-Emir, Sankt Petersburg, Expédition pour la confection des papiers d'état, 1905.

FLURY 1920, S. Flury, Islamische Schriftbänder: Amida-Diarbekr, XI. Jahrhundert, Basel, Frobenius, 1920.

GIESE 2016, F. Giese, „From Style Room to Period Room. Henri Moser’s Fumoir in Charlottenfels Castle“, in S. Costa, D. Poulot und M. Volait (Hrsg.), The Period Rooms. Allestimenti storici tra arte, gusto e collezionismo, Bologna, Bononia University Press, 2016, 153-160.

Giese/VARela Braga 2019, F. Giese und A. Varela Braga, „Translocating Metropolitan Display Strategies in 19th-century Europe: Frederick Stibbert, Henri Moser and their Orientalist Style Rooms“, International Journal of Islamic Architecture, Bd. 8, Nr. 1, 115-140, doi: 10.1386.ijia.8.1.115_1.

Hitzel 2016, F. Hitzel, „Les collections orientales d'Henri Moser“, in M. Espagne, S. Gorshenina et al. (Hrsg.), Asie centrale. Transferts culturels le long de la Route de la soie, Paris, Vendémiaire, 2016.

KLÄY 1991, E. Kläy, Orientalische Sammlung Henri Moser Charlottenfels (Bern), [Bern], Bernisches Historisches Museum, 1991.

KLÄY 1994, E. Kläy, „,Unser Ziel ist es, ein Werk zu schaffen, das uns überlebt‘. Zum wechselvollen Schicksal der Orientalischen Sammlung Henri Moser Charlottenfels“, Berner Zeitschrift für Geschichte und Heimatkunde, Bd. 56, 3, 1994, 335-358.

Moser 1886 [1885], H. Moser, À travers l'Asie Centrale: La steppe Kirghize, le Turkestan Russe, Boukhara, Khiva, le pays des Turcomans et la Perse. Impression de voyage, Paris, Plon, 1886 [Erstausgabe 1885].

Moser 1912, H. Moser, Sammlung Henri Moser-Charlottenfels: Orientalische Waffen und Rüstungen, Leipzig, Hiersemann, 1912.

Pfaff 1985, R. Pfaff, „Henri Moser Charlottenfels und seine Orientalische Sammlung“, Schaffhauser Beiträge zur Geschichte, 62, 1985, 117-156.

SAladin 1907, H. Saladin, Manuel d'art musulman, [Bd. 1]: L'architecture. Paris, Picard, 1907. SCHNELL 2009, D. Schnell, „René von Wurstemberger (1857-1935)“, Heimat heute, 2009, 4-9.

ZELLER 1915, R. Zeller, Die orientalische Sammlung von Henri Moser auf Charlottenfels: Beschreibender Katalog der Waffensammlung, Bern, Wyss, 1915.

Zeller 1922, R. Zeller, „Die orientalische Sammlung von Henri Moser auf Charlottenfels im Historischen Museum in Bern“, Das Werk, Bd. 9, 10, 1922, 189-204. 
ZeLLeR 1923, R. Zeller, Führer durch die Orientalische Sammlung Henri Moser-Charlottenfels und die Völkerkundliche Abteilung des Bernischen Historischen Museums, Bern, Grunau, 1923. 